\title{
21-cm LINE STUDIES OF THE GALACTIC CENTER REGION
}

\author{
S. CHRISTIAN SIMONSON, III \\ University of Maryland, College Park, Md., U.S.A.
}

\begin{abstract}
Features of the $21-\mathrm{cm}$ line radiation that have often been attributed to the galactic center or to activity there include, near the equator, the ' $3-\mathrm{kpc}$ arm', the 'expanding arms' at +70 and $+135 \mathrm{~km} \mathrm{~s}^{-1}$, and the 'nuclear disk', and, away from the equator, features observed by Cugnon, by Shane, by van der Kruit, and by Sanders, Wrixon, and Penzias, among others. Models of the features in the equatorial plane are discussed on the basis of the form of the $T_{\mathrm{b}}(l, V)$ contours that they generate. Both explosions in the nucleus and resonance with a spiral wave have been suggested as the cause of the 3-kpc arm and related features. Some of the models put limits on the mass distribution, particularly the amount of condensed mass at the center. Higher-latitude features have been less thoroughly modeled, but explosions in the nucleus and resonant orbits have also been suggested for their origins.
\end{abstract}

\section{Introduction}

In discussing the extensively observed $21-\mathrm{cm}$ line radiation, it seems natural to define the 'galactic center' as including the region within the inner Lindblad resonance near the radius $R=4 \mathrm{kpc}$, since features out to this distance have often been interpreted as originating near the galactic nucleus. The following subjects will be briefly reviewed here: (a) the observations and interpretations of features between $R=1$ and $4 \mathrm{kpc}$ (mainly the ' 3 -kpc arm'), (b) the same for the 'nuclear disk' at $R<1 \mathrm{kpc}$, (c) features away from the plane of the galactic equator, and (d) suggested lines of further observation and theoretical development.

\section{Features in the Galactic Plane}

The important features of the $21-\mathrm{cm}$ line profiles near the plane of the galactic equator that are thought to arise from the center region are shown in Figure 1b: (a) wide wings on the line profiles on both sides of the center, ending in an extreme-velocity ridge; (b) the well-known 3-kpc arm; (c) the 'expanding arm at $+70 \mathrm{~km} \mathrm{~s}^{-1}$, which is a rather weak and complex feature; (d) the 'expanding arm at $+135 \mathrm{~km} \mathrm{~s}^{-1}$, , about which some dispute exists concerning its exact track in the $T_{\mathrm{b}}(l, V)$ diagram; and (e) the 'nuclear disk'.

The first requirement of a theoretical interpretation of any of these features is that it reproduce the appearance of the observations. The model must therefore be put into the same form as the observations, namely, a $T_{b}(l, b, V)$ matrix giving the brightness temperature as a function of the three independent observed variables, galactic longitude and latitude and line-of-sight velocity with respect to the local standard of rest. Often some appropriate subset, such as a $T_{b}(l, V)$ contour map, is used. If the comparison with observation is satisfactory, a model can then be judged by such things as the plausibility of its symmetry properties, its energy and angular momentum requirements, or its originating mechanism. 


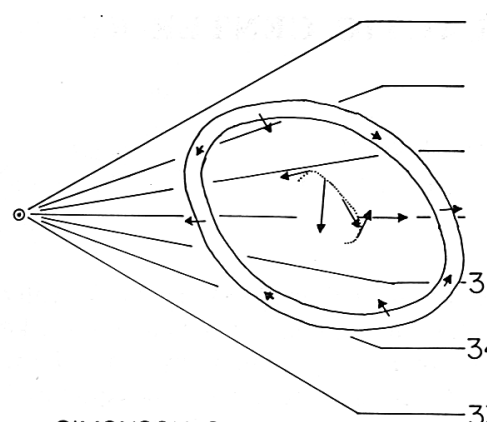

SIMONSON \& MADER

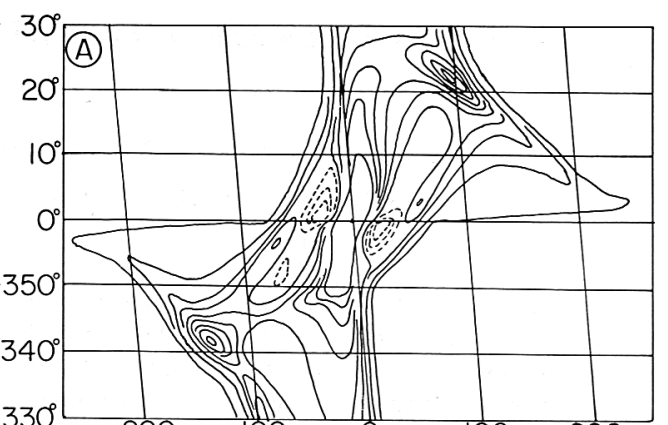

OBSERVATIONS (KERR \& HINDMAN)
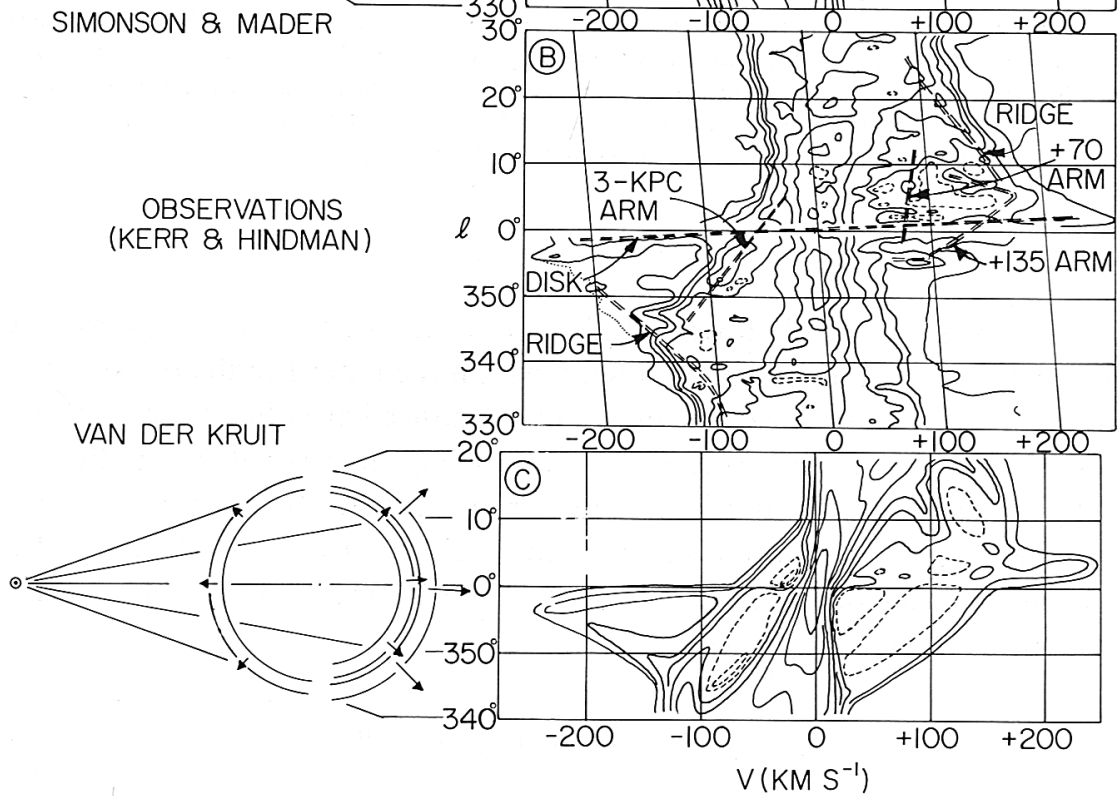

Fig. 1. $\quad T_{\mathrm{b}}(l, V)$ diagrams from (a) a model by Simonson and Mader (1973), illustrated at top left, (b) observations by Kerr and Hindman (1970), and (c) a model by van der Kruit (1971), illustrated at bottom left. Both models also contain differentially rotating disks. Arrows indicate the peculiar velocities. A schematic model for the $+135 \mathrm{~km} \mathrm{~s}^{-1}$ feature is also shown in the center of Simonson and Mader's model. Features of the observations are labeled in (b).

For the $3-\mathrm{kpc}$ arm and the $+70 \mathrm{~km} \mathrm{~s}^{-1}$ expanding arm, two alternative interpretations have been offered in suitable form for comparison: an explosion model by van der Kruit (1971) and a dispersion-ring model by Simonson and Mader (1973). The models are shown in Figure 1 together with the $T_{\mathrm{b}}(l, V)$ diagrams that they generate and the observed $T_{\mathrm{b}}(l, V)$ diagram. A third model by Sanders and Wrixon (1973), which is similar to van der Kruit's, is shown in Figure 2. Within their restrictions of simplicity, both the explosion model and the dispersion-ring model give reasonable approximations to the observations, although further refinements are desirable. Common to both models is a differentially rotating disk extending all the way in to the center. One may conclude that the velocity field between $R=1$ and $4 \mathrm{kpc}$ is pre- 
dominately that of rotation. It therefore seems possible to derive a rotation curve for this region, as has been done provisionally by Simonson and Mader, but more reliable observations are needed. The peculiar motions occur only in roughly circular structures of rather limited extent.

The choice between the two models is to be made mostly on the basis of the origin of the peculiar motions - an explosion in the galactic nucleus involving $10^{7} M_{\odot}$ and $10^{55} \mathrm{erg}$ for van der Kruit's model, or a resonance with a spiral wave whose existence is inferred from other evidence for the dispersion-ring model. One of the main difficulties with an explosion model is the short time scale of $10^{7} \mathrm{yr}$ for the presently observed phenomena. Sanders and Prendergast (1974), however, have calculated that a ring, once set in motion by an explosion, may continue to oscillate for $10^{9} \mathrm{yr}$. The energy requirements and average mass flux are still extremely large in their model $5 \times 10^{59}$ erg and $1 M_{\odot} \mathrm{yr}^{-1}$.

The expanding arm at $+135 \mathrm{~km} \mathrm{~s}^{-1}$ has so far been only roughly approximated in

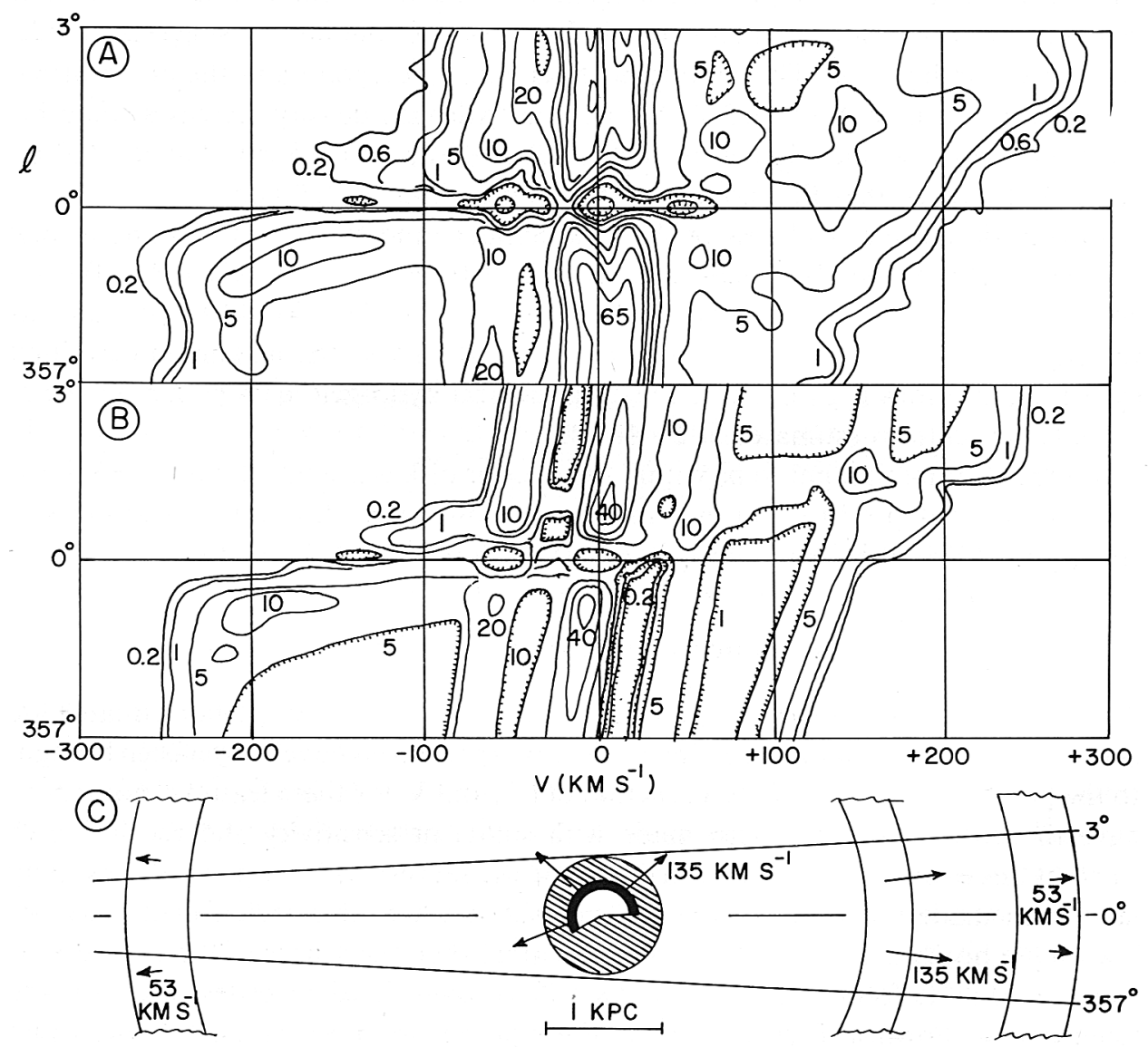

Fig. 2. $\quad T_{\mathrm{b}}(l, V)$ diagrams from (a) observations by Wrixon and Sanders (1973) and (b) a model by Sanders and Wrixon (1973), which is illustrated in (c). Contour levels are given in kelvins. 
calculations, as illustrated in Figures 1c and 2. Simonson and Mader (1973) have pointed out inconsistencies between the $T_{\mathrm{b}}(l, V)$ diagrams for expanding and rotating rings and the observations, suggesting instead a dispersion orbit interpretation as sketched in Figure 1a. Exact calculations of such an orbit await a better determination of the potential, which may include a central bar.

\section{The Nuclear Disk}

The feature in the observed $T_{\mathrm{b}}(l, V)$ diagram attributed to the nuclear disk runs diagonally from $V=-230 \mathrm{~km} \mathrm{~s}^{-1}$ at $l=358^{\circ}$ to $V=+250 \mathrm{~km} \mathrm{~s}^{-1}$ at $l=2^{\circ}$. The region within $3^{\circ}$ of the center has been newly observed by Wrixon and Sanders (1973) with the National Radio Astronomy Observatory 43-m telescope and low-noise receiver. From comparison of model $T_{\mathrm{b}}(l, V)$ diagrams with their observations, Sanders and Wrixon (1973) find evidence for pure rotational motions for $l<360^{\circ}$. They are able to place a dynamical limit of $10^{8} M_{\odot}$ on any condensed mass at the galactic center. The line radiation at $l>0^{\circ}$ is not symmetrical with that at $l<360^{\circ}$ but instead suggests a model with an expanding ring. Their model with its $T_{\mathrm{b}}(l, V)$ diagram and the observations are shown in Figure 2. At $l<360^{\circ}$ the neutral hydrogen density increases smoothly from $n=0 \mathrm{~cm}^{-3}$ at the edge of the disk, at $R=500 \mathrm{pc}$, to $n=5 \mathrm{~cm}^{-3}$ at $R<110 \mathrm{pc}$. At $l>0^{\circ}$, however, they find no neutral hydrogen within $R<300$ pc.

In considering the thickness of the disk, Sanders and Wrixon (1973) find a total thickness between points at half maximum density of $100 \mathrm{pc}$ between $R=300$ and $500 \mathrm{pc}$, in agreement with Rougoor's (1964) results. While it would require an rms velocity dispersion of $100 \mathrm{~km} \mathrm{~s}^{-1}$ to support this thickness, the maximum permitted by the observations is $24 \mathrm{~km} \mathrm{~s}^{-1}$. The total neutral hydrogen mass within $500 \mathrm{pc}$ of the galactic center is estimated at $2 \times 10^{6} M_{\odot}$.

Another feature thought to be within the nuclear disk is a small cloud at $l=359.97$, $b=-0.067$, that produces an extremely wide absorption dip at $V=+40 \mathrm{~km} \mathrm{~s}^{-1}$ (Sandqvist, 1973). It is best studied in molecular lines.

\section{Features out of the Galactic Plane}

The features of low intensity lying away from the plane of the galactic equator are shown schematically in Figure 3. Theoretical interpretations have not yet been brought to the point of calculating $T_{\mathrm{b}}$ as a function of $l, b$, and $\mathrm{V}$ for these features, nor indeed have the observations yet been made with sufficient sensitivity and resolution to warrant this effort in most cases. Furthermore, the surveys with the greatest sensitivity have been made from the Northern Hemisphere, where this region of the sky lies low on the horizon, and it is by no means certain that our census of these features is complete. In the following, the features will be considered in order of decreasing longitude as shown in Figure 3; some of the points are more extensively discussed by Simonson and Mader (1973).

(i) Shane's (unpublished) cloud at $l=8^{\circ}, b=-6^{\circ}, V=-215 \mathrm{~km} \mathrm{~s}^{-1}$, while at a 


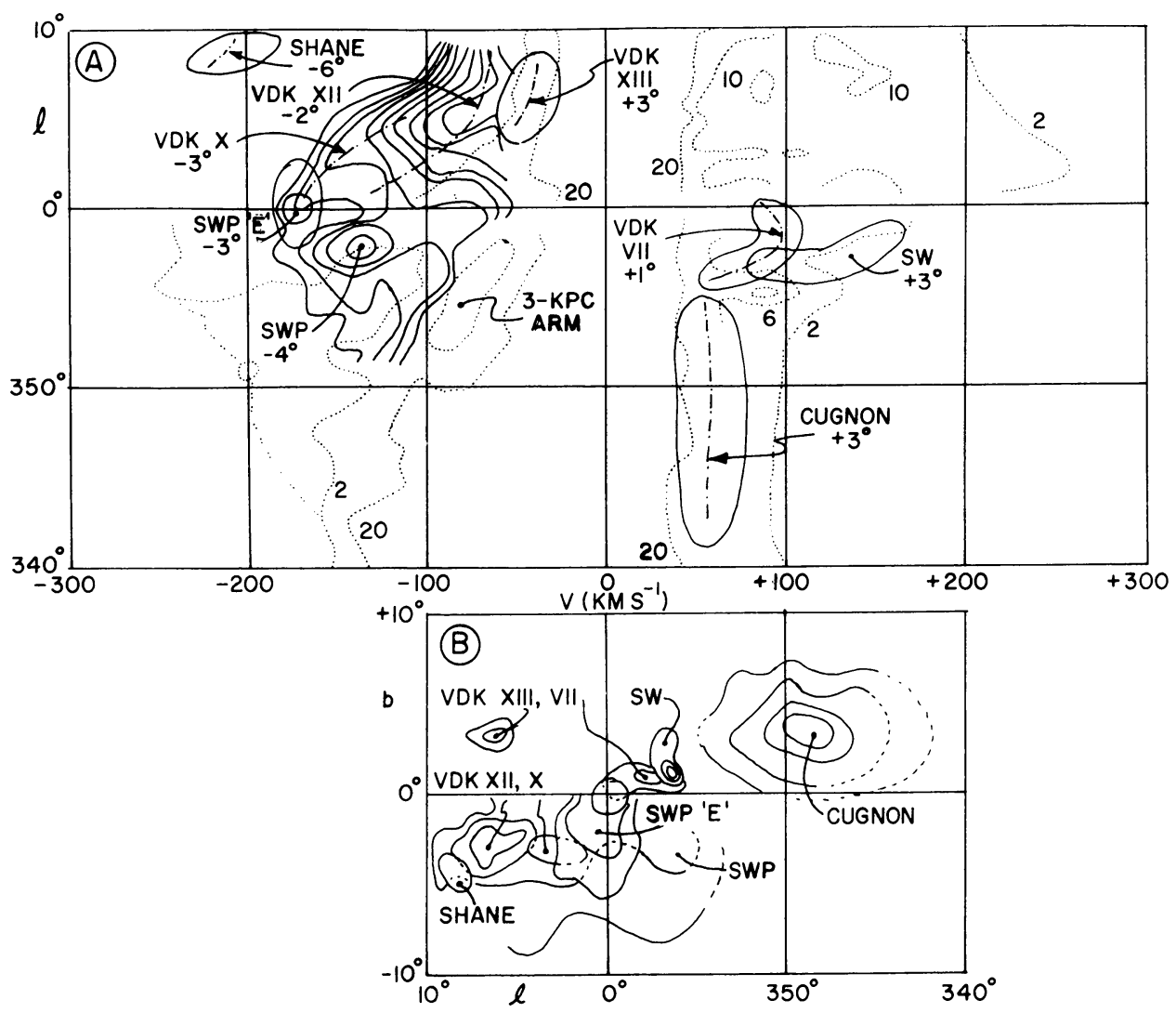

Fig. 3. (a) $T_{\mathrm{b}}(l, V)$ diagram for features out of the galactic plane, superimposed on dotted contours taken from Figure $1 \mathrm{~b}$. Representative latitudes are given. (b) $T_{\mathrm{b}}(l, b)$ diagram for the same features. Abbreviated notations: VDK = van der Kruit (1970), SWP = Sanders, Wrixon and Penzias (1972), and $\mathrm{SW}=$ Sanders and Wrixon (1972b).

position and velocity that might suggest expulsion from the center, has characteristics similar to 'high-velocity clouds' found at high latitudes. It may not be a typical feature of the center regions.

(ii) Van der Kruit's (1970) Feature XIII is the only positive-latitude feature found at negative velocities at $l>0^{\circ}$.

(iii) Van der Kruit's (1970) Feature XII and its extensions, denoted 'SWP' in Figure 3, were studied by Sanders et al. (1972) and Sanders and Wrixon (1972a). The features have been interpreted by these authors as an expanding and rotating ring. Sanders and Wrixon (1972a) suggest $v_{\text {exp }}=128 \mathrm{~km} \mathrm{~s}^{-1}, v_{\text {rot }}=151 \mathrm{~km} \mathrm{~s}^{-1}$, and $R=2.4 \mathrm{kpc}$ as characteristic parameters. They propose a connection between the negative-velocity features and the $+135 \mathrm{~km} \mathrm{~s}^{-1}$ expanding arm, the structure being viewed tangentially near $l=14^{\circ}$. Simonson and Mader (1973) suggest tracing the connection through the feature at $l=9^{\circ}, b=-0.6, V=+135 \mathrm{~km} \mathrm{~s}^{-1}$, which has more of the appearance expected of a tangential point. Davies and Cohen (in preparation) find no connection of all the various individual components into a continuous ring. but they suggest 
$v_{\text {exp }}=100 \mathrm{~km} \mathrm{~s}^{-1}, v_{\text {rot }}=60 \mathrm{~km} \mathrm{~s}^{-1}$, and $R=2 \mathrm{kpc}$ for the features at negative velocities. The non-zero rotational velocity is an important limitation on theories to account for the origin of these features. For example, van der Kruit (1971) suggests expulsion from some distance out in the nuclear disk in order to account for the angular momentum, which the feature could not have acquired by interaction with material in the galactic plane.

(iv) Feature ' $E$ ' of Sanders, Wrixon and Penzias (1972) is denoted "SWP ' $E$ '" in Figure 3. It is regarded by them as a separate feature, although there is some suggestion of a blend with van der Kruit's (1970) Feature X in Figure 3. Its position right at $l=0^{\circ}$ is suggestive of expulsion from the center.

(v) Van der Kruit's (1970) Feature VII is seen in emission in the direction of the continuum sources near the center, indicating outward radial motion.

(vi) The 'positive-velocity ridge' of Sanders and Wrixon (1972b) is denoted 'SW' in Figure 3. They suggest it is related to the $20-\mathrm{cm}$ continuum jets observed by Kerr and Sinclair (1966). However, it has characteristics very similar to those of the +135 $\mathrm{km} \mathrm{s}^{-1}$ expanding arm, which indicates that it may belong instead with the latter feature.

(vii) Cugnon's (1968) prominent feature may belong to the center region and have originated in an expulsion from the nucleus. More recent observations by Lindblad (1974), however, give somewhat more weight to one of Cugnon's alternative suggestions, namely, that it belongs to the distant, tilted-up, outer spiral structure.

Most of the explanations for the features described in this section have invoked explosions in the nucleus. If all of them so originated, it would have involved about $10^{6} M_{\odot}$ and $10^{54} \mathrm{erg}$ (van der Kruit, 1971). If only feature ' $E$ ' of Sanders et al. (1972) had been ejected, it would have taken about $10^{52} \mathrm{erg}$, according to these authors, which is quite in keeping with the scale of other activity presently observed near the center (Lynden-Bell and Rees, 1971). Alternative explanations, such as resonant orbits in the field of a central bar, deserve to be explored.

\section{Present Needs}

The state of current observations is summarized in Table I. Much observational work remains to be done. The region is difficult to observe because geometrical effects produce extremely broad and weak line profiles here. We will ultimately want to survey the entire region with the highest resolution and sensitivity achievable. The main requirements at present are more modest. The most important task is to sort out the structures in the disk. This requires close sampling between $b=-2^{\circ}$ and $+2^{\circ}$ from $l=345^{\circ}$ to $15^{\circ}$ with high angular resolution $\left(\sim 10^{\prime}\right)$, adequate sensitivity to measure profiles as weak as $1 \mathrm{~K}$, and accurately known baselines covering $600 \mathrm{~km} \mathrm{~s}^{-1}$. Only moderate velocity resolution is necessary, say $2 \mathrm{~km} \mathrm{~s}^{-1}$. Above the disk, from $|b|=2^{\circ}$ to $10^{\circ}$, the main thing is to detect all the hydrogen; its precise location is not so important as knowing how much there is. For this purpose high sensitivity and moderate angular resolution $\left(\sim 30^{\prime}\right)$ are needed, again with close sampling. 
TABLE I

Current $21-\mathrm{cm}$ line observations of the galactic center regions

\begin{tabular}{lllll}
\hline Author (date of publication) & Telescope & \multicolumn{3}{l}{ Limits and sampling interval a or resolution } \\
\cline { 3 - 5 } & & $l(\mathrm{deg})$ & $b(\mathrm{deg})$ & $V^{\mathrm{b}}\left(\mathrm{km} \mathrm{s}^{-1}\right)$ \\
\hline Burton (1970) & & & & \\
Cugnon (1968) & $25 \mathrm{~m}$ & $354(0.6) 120$ & 0 & $-340(1.7)+340$ \\
Davies and Cohen (1973) & $25 \mathrm{~m}$ & $345(1) 356$ & $+1(1)+7$ & $-50(3.4)+180$ \\
Kerr (1969) & $30 \mathrm{~m}$ and $76 \mathrm{~m}$ & $355(0.5) 10$ & $-5(0.5)+5$ & $-500(7)+500$ \\
& $64 \mathrm{~m}$ & $296(0.2) 63.5$ & 0 & $-300(7.0)+300$ \\
Kerr, Harten, and Ball (1973) & $64 \mathrm{~m}$ & $300(1) 60$ & $-2(0.2)+2$ & $-300(7.0)+300$ \\
Kerr and Hindman (1970) & $64 \mathrm{~m}$ & $270(1) 342$ & $-2(0.2)+2$ & $-150(2.1)+60$ \\
Kerr and Vallak (1967) & $64 \mathrm{~m}$ & $185(1) 63$ & 0 & $-300(7.0)+300$ \\
& & $355(0.2) 5$ & 0 & $-165(7.0)+165$ \\
van der Kruit (1970) & $25 \mathrm{~m}$ & $359(0.1) 1$ & $-1(0.2)+1$ & $-165(7.0)+165$ \\
Lindblad (1974) & $43 \mathrm{~m}$ & $350(1) 10$ & $-5(1)+5$ & $-300(10.5)+300$ \\
Menon and Ciotti (1970) & $43 \mathrm{~m}$ & $339(3) 12$ & $-10(0.3)+10$ & $-150(1)+150$ \\
Rougoor (1964) & $25 \mathrm{~m}$ & $350(0.3) 16$ & $-0.4(0.3)+0.3$ & $-264(1.7)+264$ \\
Sanders and Wrixon (1972a) & $6 \mathrm{~m}$ horn & $352(0.6) 22$ & $-0.6(0.5)+0.9$ & $-300(5)+300$ \\
Sanders and Wrixon (1972b) & $6 \mathrm{~m}$ horn & $350(2) 358$ & $-10(2) 0$ & $-340(16)-40$ \\
Sanders, Wrixon, Penzias (1972) & $6 \mathrm{~m}$ horn & $355(2) 5$ & $-5(2)+5$ & $-300(16)+300$ \\
Sandqvist (1973) & $43 \mathrm{~m}$ + moon $\{l, b=359.7,+0.46$ to & $0.35,-0.94 \mathrm{by} 0.004\}$ & $-340(16)-40$ \\
Simonson and Sancisi (1973) & $25 \mathrm{~m}$ & $356(0.6) 10$ & 0 & $-120(1.7)+120$ \\
& & $356(0.5) 2$ & $0(0.5)+5$ & $-120(3.4)+120$ \\
& & $10(0.5) 24$ & $-8(0.5)+5$ & $-60(3.4)+180$ \\
& & $10(0.5) 250$ & $-10(0.5)+10$ & $-140(2.1)+240$ \\
Weaver and Williams (1973) & $26 \mathrm{~m}$ & $11(0.2) 233$ & $-2(0.2)+2$ & $-60(2)+180$ \\
Westerhout (1973) & $91 \mathrm{~m}$ & $357(0.3) 3$ & $-3(1)+3$ & $-500(5.5)+500$ \\
Wrixon and Sanders (1973) & $43 \mathrm{~m}$ & & $-1(0.3)+1$ & \\
& & & &
\end{tabular}

a Sampling interval given when it exceeds beamwidth.

b Velocity limits usually vary with longitude; the range near $l=0^{\circ}$ is tabulated.

Theoretical developments to be hoped for center mostly about mechanisms. The models calculated until now have been largely kinematical. For dispersion rings, while individual resonant orbits have been calculated, collective effects have yet to be included. Hopefully, this would provide values for the peculiar velocity amplitudes and sizes of the structures. There is also the question of motions in the field of a possible central bar. Gas dynamics must be treated along with the stellar dynamics. Explosive theories also suffer from lack of definite values and must, in addition, account for the prominent asymmetries that are observed.

\section{References}

Burton, W. B.: 1970, Astron. Astrophys. Suppl. 2, 261.

Cugnon, P.: 1968, Bull. Astron. Inst. Neth. 19, 363.

Davies, R. D. and Cohen, R. J. : 1973, private communication.

Kerr, F. J.: 1969, Australian J. Phys. Astrophys. Suppl., No. 9, 1.

Kerr, F. J. and Hindman, J. V.: 1970, Australian J. Phys. Astrophys. Suppl., No. 18, 1.

Kerr, F. J. and Sinclair, M. W.: 1966, Nature 212, 166.

Kerr, F. J. and Vallak, R.: 1967. Australian J. Phys. Astrophys. Suppl., No. 3, 1. 
Kerr, F. J., Harten, R. H., and Ball, D. L.: 1973, Astron. Astrophys. Suppl., in preparation.

Kruit, P. C. van der: 1970, Astron. Astrophys. 4, 262.

Kruit, P. C. van der: 1971, Astron. Astrophys. 13, 405.

Lindblad, P. O.: 1974, Astron. Astrophys. Suppl. 16, 207.

Lynden-Bell, D. and Rees, M.: 1971, Monthly Notices Roy. Astron. Soc. 152, 461.

Menon, T. K. and Ciotti, J. E. : 1970, Nature 227, 579.

Rougoor, G. W.: 1964, Bull. Astron. Inst. Neth. 17, 381.

Sanders, R. H. and Prendergast, K. H.: 1974, Astrophys. J. 188, 489.

Sanders, R. H. and Wrixon, G. T.: 1972a, Astron. Astrophys. 18, 92.

Sanders, R. H. and Wrixon, G. T.: 1972b, Astron. Astrophys. 18, 467.

Sanders, R. H. and Wrixon, G. T.: 1973, Astron. Astrophys. 26, 365.

Sanders, R. H., Wrixon, G. T., and Penzias, A. A.: 1972, Astron. Astrophys. 16, 322.

Sandqvist, Aa.: 1973, Astron. Astrophys. Suppl. 9, 381.

Shane, W. W.: 1972, Astron. Astrophys. 16, 118.

Simonson, S. C. and Mader, G. L.: 1973, Astron. Astrophys. $27,337$.

Simonson, S. C. and Sancisi, R.: 1973, Astron. Astrophys. Suppl. 10, 283.

Weaver, H. and Williams, D. R. W.: 1973, Astron. Astrophys. Suppl. 8, 1.

Westerhout, G.: 1973, Maryland-Green Bank Galactic 21-cm Line Survey, 3rd edn.. Univ. of Maryland,

College Park, Maryland.

Wrixon, G. T. and Sanders, R. H.: 1973, Astron. Astrophys. Suppl. 11, 339.

\author{
S. Christian Simonson, III \\ Astronomy Program, \\ University of Maryland, \\ College Park, Md. 20742, U.S.A.
}

\title{
DISCUSSION
}

Menon: There is a tendency in the literature to imply that all radial motions in the galactic plane cease with the '3-kpc expanding arm'. However, it has been known for some time that radial motions at slightly reduced velocities persist to at least $5 \mathrm{kpc}$. Hence in discussing the energies and ages of the phenomena involved we should discuss motions at least up to $5 \mathrm{kpc}$ from the center.

Simonson: In our model, the motions extend up to 4.7 or even $5 \mathrm{kpc}$ from the center.

Kahn: You mentioned the possibility that there might be a bar at the galactic center. If this bar makes an appreciable contribution to the gravitational field, the system would no longer have axial symmetry. Will this help with your angular momentum problem?

Simonson: It may help to organize the gas into resonant orbits, but orbits in a $\theta$-dependent potential are hard to calculate.

Pishmish: I would like to comment on the rotating and expanding ring structure which is proposed to explain the kinematics of the molecular clouds in the central region of the Galaxy. It seems to me that the hypothesis of a ring is an oversimplified one. If one accepts that the expansion is a result of mass ejected from a rotating nucleus, the locus of the ejected matter will not be a circle but a spiral. The pitch, of course, depends on the velocity of expansion and the velocity of rotation. It appears that it is easier to produce a spiral than to produce a ring, as the latter would require an isotropic expulsion of matter, a phenomenon much more difficult to explain than a localized ejection. The formation of such a spiral locus was discussed by Huang and Pishmish back in 1960 (Bol. Obs. Tonantzintla and Tacubaya, No. 19).

Simonson: In fact, if you want to adopt an explosion model for the $+135 \mathrm{~km} \mathrm{~s}^{-1}$ feature, some such thing as that would be probably all that is necessary, so that it is not a ring but has more of a spiral shape. Both models for the 3-kpc arm show expansion when one looks toward the center but circular velocities at an angle from the center, so both velocities are probably present, but it is hard to get the right match.

Oort: You mentioned the possibility of a bar in the central part. Don't you think this would be difficult to reconcile with this very strongly differentiately rotating nuclear disk that you find in the center? The gravitational field wouldn't seem suitable for a bar at all.

Simonson: In view of the fact that the nuclear disk is asymmetrical north and south, that material might not be exactly in the form of a nuclear disk but have properties resembling resonant orbits in the 
field of a bar. I'm not prepared to do any more than just raise the suggestion at this point. We need higher angular resolution there.

Baldwin: Do you imply that some changes are necessary in the inner parts of the galactic rotation curve or the distribution of mass?

Simonson: Schmidt took no observed points between $R=1$ and $4 \mathrm{kpc}$ but used just a point mass at the center. We find his curve to be about $15 \%$ low, reaching a minimum of $185 \mathrm{~km} \mathrm{~s}^{-1}$, at $R=2 \mathrm{kpc}$, while observations suggest $210-215 \mathrm{~km} \mathrm{~s}^{-1}$ as the minimum at $R=3-4 \mathrm{kpc}$. Adding a Gaussian disk of mass $4 \times 10^{8} M_{\odot}$ and radial dispersion $1 \mathrm{kpc}$ to Vandervoort's (Astrophys. J. 161 (1970), 67) model would about do it.

Burke: One way of avoiding the angular momentum problem inherent in explosion models of the galactic center is to assume that the explosion provides the energy (by a beam of relativistic particles, for example) but not much mass or momentum. The matter and the momentum is that of the gas in the disk. which would be partly sprayed up above the plane by the explosion, but preserving its angular momentum.

Churchwell: In the explosion models so far proposed which require energies of $10^{55}-10^{59} \mathrm{erg}$ it seems to me that the gas would have to be ionized; however, what is observed is neutral hydrogen. What is the time scale for recombination for the expelled high velocity Hi clouds?

Simonson: In van der Kruit's model, the gas starts off ionized but cools rapidly on a time scale of $10^{6} \mathrm{yr}$; we see it now recombined after $10^{7} \mathrm{yr}$.

Davies: Observations of $\mathrm{HI}_{\mathrm{I}}$ in the galactic center $\left(l=5^{\circ}\right.$ to $+10^{\circ}, b=-5^{\circ}$ to $\left.+5^{\circ}\right)$ have been made at Jodrell Bank with the Mark II (32' beamwidth) and Mark IA (12') telescopes. These observations were made at high sensitivity ( $r m s$ noise $\sim 0.1 \mathrm{~K}$ ) to provide new information on the structure of the galactic center region. The full angular resolution and sensitivity were necessary to delineate many of the galactic center features. 